\title{
ON THE STROUHAL NUMBER OF FISH UNDULATORY SWIMMING: A SIMPLE PREDICTIVE ANALYSIS BASED ON THE BUCKLING OF COLUMNS
}

\author{
J.G. CERVANTES, F. MÉNDEZ \& F.J. SOLORIO \\ Faculty of Engineering, National University of Mexico, Mexico.
}

\begin{abstract}
An analogy between fish undulatory swimming and the buckling of columns is presented and discussed. The most important aspects of the undulatory swimming of a fish, from the mechanical point of view, are described. The buckling response of solid and fluid columns and its mathematical modelling are summarized and applied to a swimming fish, as an analogue of buckling columns. The predictable Strouhal number for a buckling fish agrees well with the apparently universal experimental value observed by numerous investigators of fish undulatory swimming.

Keywords: buckling of columns, fish undulatory swimming, fluid columns, hydrodynamic foils, solid columns,
\end{abstract} Strouhal number.

\section{INTRODUCTION}

Long et al. [1] quoted in the heading aphorism of their article that '.. in spite of being heterogeneous in structure, the body of the fish is bending as a continuous beam', [2]. According to the former authors, detailed measurements of in vivo axial strain of muscles have shown that the swimming body of many fish species bends like a homogenous, continuous beam (Fig. 1). This simple beamlike behaviour is surprising because the underlying tendon and muscle structure and their behaviour are complex. Indeed, many fish species swim by undulating their bodies, resembling a continuously buckling column, and their shape very much corresponds to a solid massive tapered column (Fig. 2).

In the present investigation, a simple and direct way to predict the waveform and the wavelength of the undulatory swimming of a fish is presented. The proposal consists of a global analogue of the steady-state undulating swimming fish as if it was a solid tapered column under reverse buckling conditions. This reverse buckling effect essentially means that the steady-state moving column is deforming due to the internal action of the muscles and tendons, giving rise to a longitudinal force whose reaction opposes and overcomes the hydrodynamic forces and results in a net thrust. The analogue permits the prediction in a simple manner of the Strouhal number which is characteristic of the undulatory swimming and agrees well with the experimental results reported in the open literature.

The article is organized as follows. First, the basic aspects of undulatory swimming are reviewed. Next, the most important results of the buckling analysis of solid columns are outlined. Their implications to predict buckling Strouhal numbers of fluid columns as an analogue to solid columns are briefly revisited. Finally, the extension of this approach to a fish during dynamic equilibrium undulatory swimming as a highly flexible tapered column is described and discussed.

\section{FISH UNDULATORY SWIMMING}

The archival literature related to the propulsion of aquatic animals is vast and reflects the importance of this topic for Life Sciences and Applied Mechanics. The swimming of fish and cetacean species under different circumstances represents an essential element in adaptation to their environment and 
constitutes a complex physical problem that only in the past two decades has been accomplished with enough detail by authoritative contributions to this topic [3-13]. Besides its obvious scientific interest, fish locomotion is important for the characterization of exploitable species by fisheries, and has also been considered as an alternative mechanism for the conception and design of autonomous underwater vehicles.

Fish swimming modes have been recently reviewed and classified by Sfakiotakis et al. [13], based in the propulsion element and in the form of movement used by a fish to generate thrust. A large portion of fish species and the cetacean mammals swim by means of the undulatory bending of their whole body, an action that propagates from head to tail, producing the necessary thrust force to counterbalance friction and added mass from the water (Fig. 1). These buckling travelling waves are

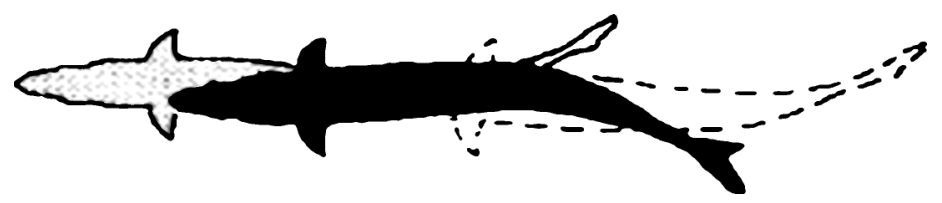

Figure 1: Undulatory swimming fish (taken from [13]).

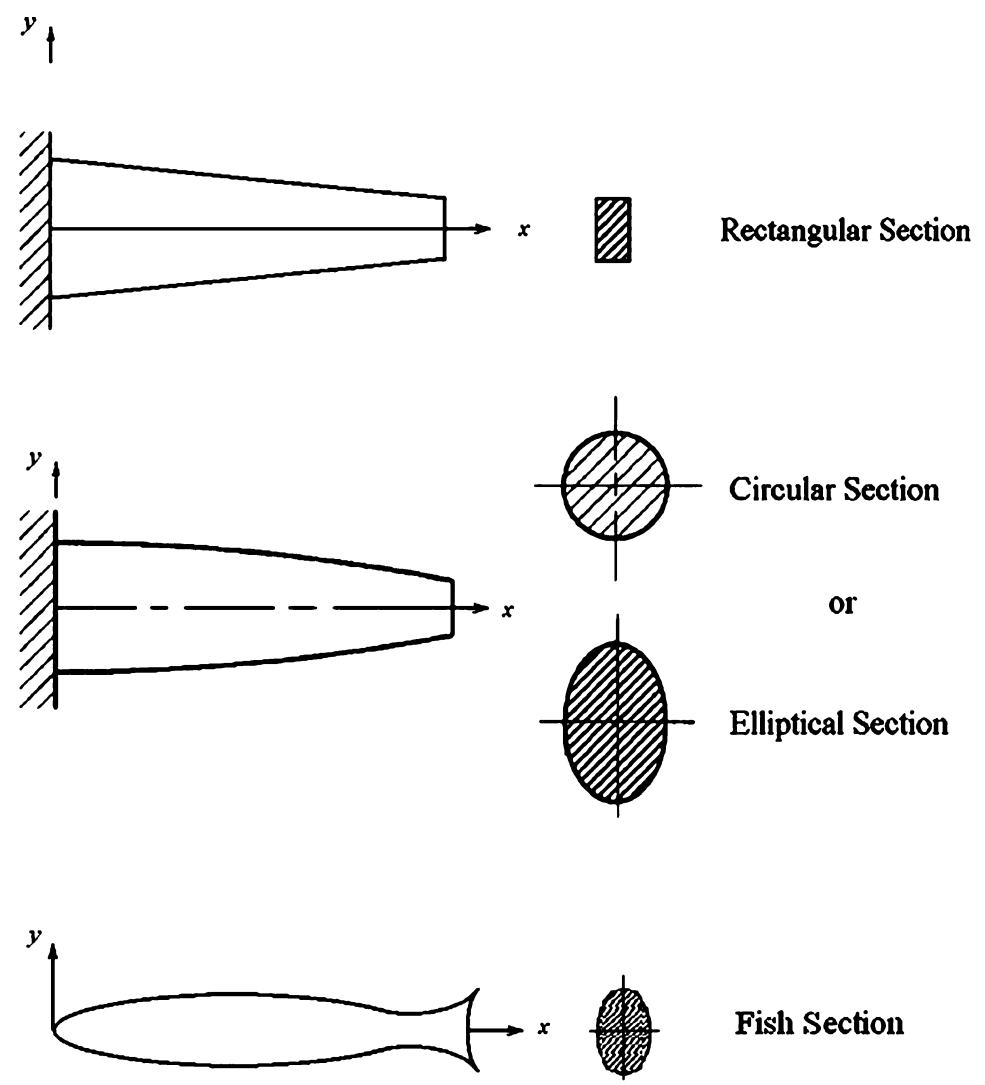

Figure 2: Examples of tapered columns. 
produced by the fish muscles, tendons and bone structure that alternatively stretch and restrain the axial and transverse structures of the body. According to Blight [14], the precaudal region of the body of a fish operates as a wave generator.

A fish is a body with a hydrodynamic shape very similar to a tapered column with large slenderness ratio (Fig. 2). Its locomotion is the result of the unbalanced internal and external forces on the body, composed mainly by the contraction and extension of muscles of the fish and the reaction to the external flow (drag and added mass). Many fish species swim by generating undulatory waves that propagate from head to tail, transferring momentum to the surrounding water, and producing thrust. By changing the shape and speed of these travelling waves, they modulate the rate of work production and hence control swimming speed [9].

The balance of all forces that act at any time in every element of the fish - internal forces from the apparent viscoelasticity of the tissues in the back-and-forth undulatory or stretch-restrain motion, and the hydrodynamic and inertial external forces - provoke the thrust necessary for the swimming of the fish, either in steady-state or in transitional motion. At any instant, the integration of these forces around the body gives rise to either acceleration or deceleration of the fish, not only in the forward direction but also laterally and rotationally.

As part of the fish and surrounding water interaction, the forces due to the fluid pressure and the total drag act on the fish body surface. To know the distribution of these reaction forces, it is necessary to integrate the Navier-Stokes equations for the ambiance flow subject to the boundary conditions that impose the undulating fish surface and taking into account any flow characteristics alien to the fish.

The dynamical analysis of the fish body implies the consideration of all the forces together with the associated bending moments giving rise to a form of the wave equation if small oscillations are considered. This equation represents a complex dynamic system composed by (a) a source of energy and power (the fish metabolism) that manifests itself through the internal forces (contraction and extension of muscles); (b) a stiffness/damping constitution (viscoelasticity of internal tissues); (c) the reaction to the external flow (drag and added mass); and (d) the resulting fish rectilinear motion either, uniform or accelerated, depending on the force balance. It represents a formidable problem of mechanics since it implies a complex interaction of the living organism with the external flow. The kinematical characteristics of the fish body are unknown beforehand and are part of the solution. The problem has been only partially solved through various methods and techniques $[6,7,15-18]$, but many aspects remain insufficiently known or understood.

The oscillating tail of the fish serves as a tuned amplifier of the wave, generating a thrust-producing jet instead of a drag-producing wake behind the fish [7]. The counter-rotating vortices arrangement at the wake of the fish is the result of a controlled emission of these structures by the oscillatory tail of the specimen. The fish tail takes advantage of the buckling undulating motion of the body to recover as much energy as possible of the moving body, and to change the drag properties of a wake to a thrust producing jet flow. As a consequence of the undulating body of the fish during its rectilinear translation and the lateral motion of its tail, there is a peculiar wake behind the fish. The average velocity distribution in the wake behind the streamlined profile of a fish has the shape of a penetrating jet flow. This jet is unstable, in such a way that being excited harmonically by the oscillating fish body, an array of vortices is formed with reversed rotation respect to the classic von Karman street (Fig. 3). The frequency of formation of these vortices lies within the frequency range - relatively narrow - of maximum amplification of a perturbation of the jet flow, according to hydrodynamic stability theory [7, 20]. It is well known that a foil oscillating in heave and pitch can produce a mean forward thrust force, through the generation of a reversed vortices street. The average thrust can be estimated based on the momentum flux associated with the jet profile $[19,21]$. The efficient development of the thrust force is associated with the vortices structure: the frequencies of maximum growth must correspond to the higher 


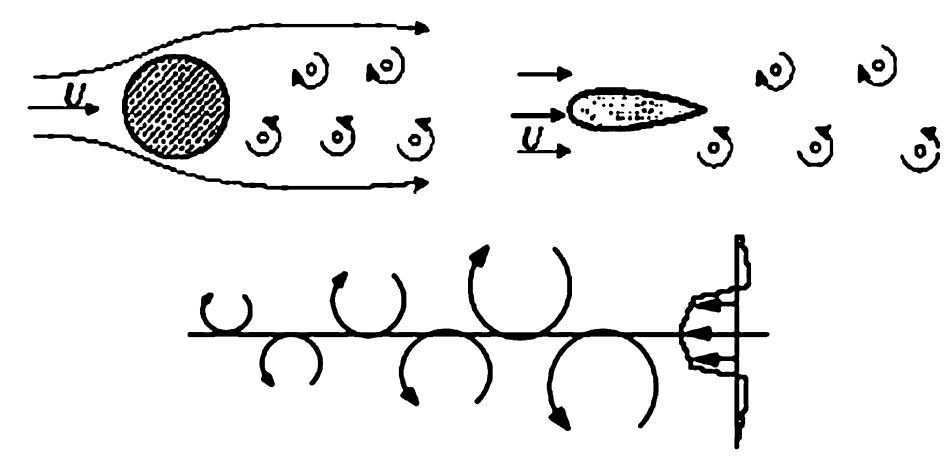

(a)
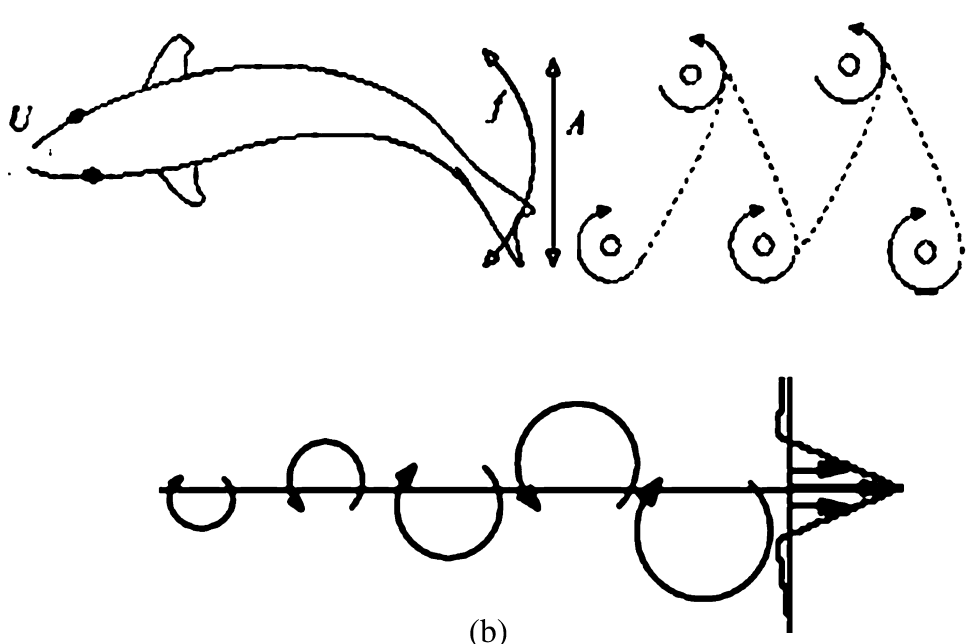

(b)

Figure 3: Vortex wake behind a submerged body: (a) drag-inducing velocity profile in a still cylindrical or hydrodynamic shape; (b) thrust-inducing velocity profile in a flapping fish (adapted from [13, 19]).

efficiency of the thin profile, as these result from the most expedient formation of the vortices [7]. In the words of these authors, 'the existence of preferred frequencies in the wake gives a new dominant parameter for the fish propulsion, the Strouhal number . . . These frequencies correspond to the Strouhal numbers in the range $0.25-0.35$, with a most likely value of 0.3 . The Strouhal number is defined as $\mathrm{St}_{\mathrm{f}}=f A / V$, where $f$ is the frequency of the tail oscillations, $A_{\mathrm{t}}$ is the total width of the wake, taken as the maximum excursion of the tail (i.e. a double amplitude), and $V$ is the mean forward velocity of the fish. These authors have recorded many data, including their own experimental results in a wide compilation of fishes and cetaceans, which verify the proposed theory. In a more recent article, Taylor et al. [22] verified and extended the above-summarized statements for many aquatic and avian species and confirmed the optimal range $0.2<\mathrm{St}_{\mathrm{f}}<0.4$ expected for an efficient cruise propulsion.

In summary, it can be said that many fish species swim by passing waves of bending down their bodies. The dynamical analysis of this undulatory swimming has been approached from different standpoints, but its full account has not been accomplished. For instance, to the best knowledge of the authors of the present investigation, the totality of the articles published in the archival literature 
dealing with the mathematical modelling of fish swimming invariably assume (a priori) a known sinusoidal shape for the undulatory motion, based on numerous experimental results that seem to confirm this behaviour.

In this article, a simple and direct way to predict the waveform characteristics of the fish undulatory swimming is presented. The procedure is based on the analogy that can be discovered between the undulatory swimming of a fish and the buckling response of a solid column, when the latter is compression loaded at its extremes. The fish body nevertheless, is made out of several materials like in a composite structure. This means that it is not only elastic, but has also viscous and flowing properties. The inner structure of a fish is a viscoelastic complex, with a variety of qualities, forms and functions, and therefore it is very difficult to model from a constitutive point of view [15, 23, 24]. Seen in a general perspective, the fish materials are somewhere between solids and fluids, with properties that combine both kind of states. It is important then, to bring in the following sections, the parallel consideration of solid and fluid buckling columns.

\subsection{Uniform cross-section solid columns}

\section{THE BUCKLING OF SOLID AND FLUID COLUMNS}

The instability process (or buckling) associated with elastic solid columns and other structural elements has been widely studied since it was first considered by Euler in his pioneering investigations. It consists of a new equilibrium condition that a solid structure attains under compression loads, characterized by a permanent flexion strain in a more stable state than the one it had before [25].

Consider the equilibrium conditions for a solid column of uniform cross section (Fig. 4). The column simultaneously experiences axial compression and bending moments as a result of external forces acting at both ends (the Euler-Bernoulli beam). Static equilibrium means that there is a balance of the axial compression forces, $C$. It also means that rotational equilibrium has to be preserved from a section where the bending moment is $M_{0}$ to any section where the bending moment is $M(x)$. In the most general case, the compression forces $C$ are not collinear due to any small lateral deflection in the column, $h(x)$. The bending moment $C h$ from the eccentricity $h$ of the compression force must be balanced at all times by the cross-sectional bending moment $M(x)$, giving rise therefore, to the Euler buckling equation:

$$
-M(x)+C h(x)+M_{0}=0 .
$$

The analysis of a linear strain related to pure bending gives as a result, that the bending moment in any section of the column is proportional to the curvature of the column; so in the case of an elastic column,

$$
M(x)=\frac{E I}{R_{\infty}}=-E I \frac{\mathrm{d}^{2} h}{\mathrm{~d} x^{2}},
$$

where $E$ is the elasticity modulus, $I$ is the area moment of inertia of the cross section, and $R_{\infty}$ is the radius of curvature. Substituting eqn (2) in eqn (1), the balance equation reads as a constant coefficients linear equation of the form

$$
\frac{\mathrm{d}^{2} h(x)}{\mathrm{d} x^{2}}+\frac{C}{E I} h(x)=\text { constant },
$$




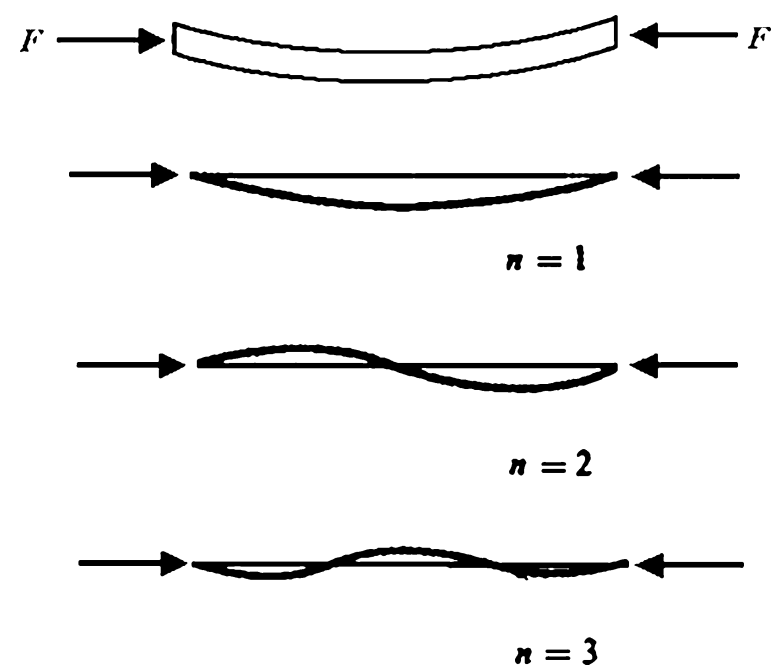

Figure 4: Buckling of a uniform solid column showing eigenvalue modes.

where $m=(C / E I)^{1 / 2}$ leads to the eigenvalues of the problem in the case of a uniform cross-section column (Fig. 4). The eigenfunctions that form the solution - sine or cosine functions - depend on the boundary conditions of the problem.

\subsection{Tapered solid columns}

Of interest to the undulatory swimming of a fish, is the tapered column (Fig. 2). This non-uniform cross-section column corresponds to many optimal shapes found both, in nature and in engineering and architecture applications. Tapered structural members for instance, are widely used in structural frames to make the stresses more evenly distributed and to reduce at the same time the consumption of the material [26]. They have also been used to fit many architectural elements in old houses and contemporary buildings. There are on the other hand, many examples in living organisms, both vegetal and animal, where beams and column elements have a tapered shape to compromise stiffness with the use of materials to have optimal resistance and performance for given load conditions [27, 28].

The Bernoulli-Euler equilibrium equation for small deflections eqn (3) in the case of a tapered column is given by [29, 30],

$$
\frac{\mathrm{d}^{2} h(x)}{\mathrm{d} x^{2}}+\frac{C}{E I(x)} h(x)+M_{0}=0
$$

where the area moment of inertia $I(x)$ is now a function of the longitudinal coordinate $x$. This means that eqn (4) is a second-order differential equation with variable coefficients. Its general solution depends on the form of the coefficient of the second term in the equation and can be from simple sine functions obtained by direct integration, to numerical solutions full of complexities obtained through advanced structural analyses methods, depending on the shape of the tapered column. For plain forms resembling the shape of a fish, simple but significant solutions can be obtained as presented later in this article. 


\subsection{Fluid columns}

Buckling phenomena have also been considered in fluid currents under various conditions [31-35]. In analogy with a solid column, a segment of an unconfined fluid current can be visualized, in a standstill image of the moving fluid, as if instantaneously was a solid column subject to compression forces and bending moments at its ends, i.e. at the flow inlet and outlet cross sections of the volume control depicted in Fig. 5, giving rise to a buckled fluid column. Examples abound from very viscous fluid columns [35], to low viscosity flows [33] and free turbulent shear flows [34]. In the case of a solid column, the forces are given by the loads applied to the column and the bending moments originate from the boundary conditions at the supports. The uniform solid column can exhibit multiple deformations, corresponding to the eigenvalue modes of the elastic response to the force balance. In the case of the fluid column, the axial loads correspond to the momentum fluxes at the flow inlet and outlet, and the bending moments arise from the eccentricity of the axial loads. The strain in this case consists of a unique mode, and is associated with the single frequency lateral oscillations that are typical in these kinds of flows.

The buckling fluid column is therefore the analogue, in continuous movement, of the buckled solid column: the fluid column is dynamic (flow, lateral oscillations), whereas the solid column is static (load, shear in rest). Buckling phenomena can be considered as the response of many slender mechanical elements, to axial longitudinal loading at its extremes. These mechanical elements are continuous media, and can be elastic solids or Newtonian fluids as well as flows with very little viscosity like turbulent free shear flows, that exhibit their incompressibility - or impossibility of internal structure accommodation - by means of lateral deformations in the case of solids, or single frequency oscillations in the case of fluids. The geometric or kinematic characteristics of either case are predictable if the amplitude is small.

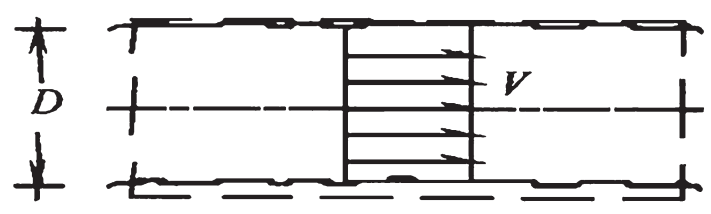

(a)

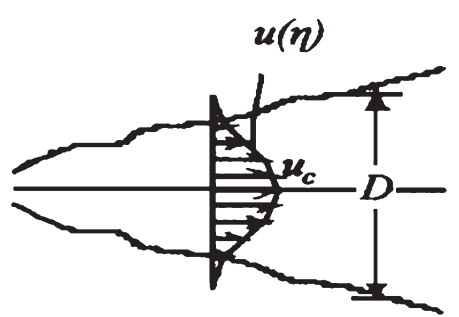

(b)

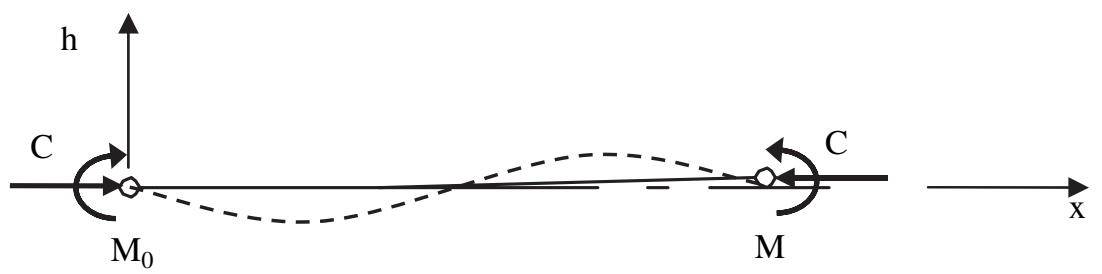

(c)

Figure 5: Buckling fluid columns: (a) uniform cross-section and velocity profile; (b) variable crosssection and velocity profile; (c) coaxial compression forces and bending moments induce buckling. 
As indicated in Fig. 5, Bejan [33] considered the equilibrium conditions for a segment of a fluid column analogue to the solid column in Fig. 4, as

$$
\left(\rho V^{2} I\right) \frac{\mathrm{d}^{2} h}{\mathrm{~d} x^{2}}+\left(\rho V^{2} A\right) h+M_{0}=0
$$

where $\rho V^{2} I$ and $\rho V^{2} A$ now represent the bending stiffness and the compressive force on the fluid column, respectively. This equation has the form given in eqn (3), but its solution is represented by a sine function with a single wavelength. This is due to the fact that the compressive force and the elasticity modulus in the case of the fluid column - the coefficients of the first two terms of eqn (5) - are proportional to each other. The obtained wavelength is

$$
\lambda_{\mathrm{B}}=2 \pi \sqrt{\frac{I}{A}}
$$

and an order of magnitude estimate of 0.5 for the buckling Strouhal number, defined as

$$
\mathrm{St}_{\mathrm{B}}=\frac{D}{\lambda_{\mathrm{B}}},
$$

for different flow configurations, was obtained.

Cervantes et al. [34] completed the above-mentioned analysis under the same concepts and principles for a more general case of fluid column as can be identified in various cases of turbulent free flows. They took into account in their analysis the non-uniform velocity profiles and the varying cross section along the flow direction, as reported in the archival literature, typical of this kind of flow. Moreover, based on concepts derived from the second law of thermodynamics, these authors explained the marginal differences that appear for the Strouhal number in various analysed cases.

The balance equation obtained for this more general case is

$$
\frac{\mathrm{d}^{2} h(x)}{\mathrm{d} x^{2}}+\frac{F_{2}(x)}{F_{1}(x) D^{2}(x)} h(x)+M_{0}^{\prime}=0 .
$$

Again this equation has the same form as eqn (3), but this time the coefficient of the second term includes two integral expressions $\left(F_{1}\right.$ and $\left.F_{2}\right)$ that take into account the velocity profile, the cross-section area and the area moment of inertia, as functions of the longitudinal space coordinate through the use of similarity variables. (See Cervantes et al. [34] for more details on this formulation.)

The single wavelength of the solution of eqn (8) for this general case is given by

$$
\lambda_{\mathrm{B}}=2 \pi \sqrt{\frac{F_{1} D^{2}}{F_{2}}} .
$$


For the case where the fluid velocity profile is uniform and the cross section is constant along the flow (the so-called 'top-hat' profile), eqn (9) simplifies to eqn (6).

The dimensionless wavelengths of turbulent jets and wakes that have an oscillatory behaviour were calculated using eqn (9), with velocity profiles amply documented in the literature. Table 1 includes a summary of these results under the subheading 'Fluid columns'. As seen, almost the same value is obtained for the calculated dimensionless wavelengths for all the considered cases, despite that they correspond to different flow configurations. They confirm the order of magnitude of 0.5 estimated by using eqns (6) and (7), and agree with the experimental measurements of the natural frequency of turbulent jets exposed to a range of external excitation frequencies, as reported by Bejan [33] who considered this value as a universal constant. The slight differences among them are due to the different degree of spreading of the corresponding profile, giving rise to different values of the integrals $F_{1}$ and $F_{2}$ in eqn (9), and can be explained on thermodynamic grounds [34, 38].

In summary, solid and fluid columns buckle alike when subjected to axial loads. Uniform crosssection solid columns respond with multiple sinusoidal modes, corresponding to the eigenfunctions of the solution of a small deflections linear model. Simple tapered solid columns are characterized by a single wave solution whose amplitude is a function of the longitudinal coordinate, as a result of the axial coordinate dependence of the area moment of inertia. Fluid columns, on the other hand,

Table 1: Strouhal number for various fish, foils and columns.

\begin{tabular}{lccc}
\hline & & \multicolumn{2}{c}{ Strouhal number } \\
\cline { 3 - 4 } Description & $\begin{array}{c}\text { Characteristic } \\
\text { length }\end{array}$ & $\begin{array}{c}\text { Buckling (predicted) } \\
\mathrm{St}_{\mathrm{B}}=D / \lambda_{\mathrm{B}}\end{array}$ & $\begin{array}{c}\text { Flapping (measured) } \\
\mathrm{St}_{\mathrm{f}}=f A / V\end{array}$ \\
\hline Fish and cetaceans & & & \\
$\quad$ Sunfish & $2 b_{0}$ & 0.37 (eqn (13)) & \\
Sunfish & $2 b_{0}$ & $0.3-0.6$ (eqn (13)) & $0.25-0.38[7]$ \\
Various species & $A$ & & $0.2-0.4[22]$ \\
Various species & $A$ & & $0.3[12]$ \\
$\quad$ Dolphin & $A$ & & $0.3[21]$ \\
Hydrodynamic foils & & & $0.25-0.4[19]$ \\
Wake of oscillating airfoil & $2 b$ & 0.3 [22] & \\
Oscillating foil & $2 b$ & & \\
$\quad$ (high efficiency) & & & \\
Oscillating foil & $2 b$ & & \\
$\quad$ (optimal thrust) & & & \\
Solid columns & & & \\
Circular & $D$ & 0.636 (eqn (6)) & \\
Rectangular & $D$ & 0.552 (eqn (6)) & \\
Ellipsoidal & $2 b_{0}$ & 0.318 (eqn (6)) & \\
Fluid columns & & & $0.483[36]$ \\
'Top hat' plane jet & $2 b$ & 0.552 (eqn (9)) & \\
Turbulent plane jet & $2 b$ & 0.575 (eqn (9)) & \\
Turbulent round jet & $D$ & 0.588 (eqn (9)) & \\
Turbulent plane wake & $2 b$ & 0.546 (eqn (9)) & \\
Wakes behind cylinders & $D$ & & \\
\hline
\end{tabular}


always buckle with a single waveform due to the proportionality of the bending moment and the pseudoelasticity modulus, and also due to the similarity structure of their velocity profiles, no matter the cross-section variation along the longitudinal coordinate.

\section{SWIMMING FISH: A MOVING INVERSE BUCKLING COLUMN}

The steady-state undulating swimming fish can be seen as if it was a tapered column undergoing a reverse buckling process. In a solid column, when compressive external forces and bending moments are applied at its ends, lateral displacements of the column may arise (buckling). In a similar but inverted way, the body of a fish undulates (bends) as a result of the complex action of its muscles, tendons and bones. The lateral bending wave that travels along its entire length produces the force that acts on the surrounding water. The corresponding reaction force consists of the net thrust that pushes the fish through the water (Fig. 6). In other words, the coaxial forces in a solid column give rise to the column buckling, whereas the undulatory movement of a fish generates the axial force whose reaction from the water pushes the fish through the water. For this reason, the whole effect can be properly considered as an inverse buckling.

The analysis presented in this investigation, considers the steady-state undulatory swimming fish in dynamic balance of the forces acting on it. The overall rectilinear motion at constant velocity of the fish (no acceleration) means that there is a dynamic equilibrium among internal actions (muscle and tendon), external hydrodynamic forces (drag and added mass) and net reaction (thrust). The thrust is the longitudinal reaction to all the forces acting on the fish, especially those generated by the bending waves originating from the internal muscle and tendon actions throughout the fish body. In the reverse buckling conception herein presented, this internal resultant force globally acts in tension along the fish, thus creating the continuously bending waves along the body. The internal resultant force exerts a bending moment due to its eccentricity with respect to the centre line of the body. This force moment must be balanced at all times by the cross-sectional bending moment in equilibrium conditions, giving as a result a linear ordinary second-order differential equation, if
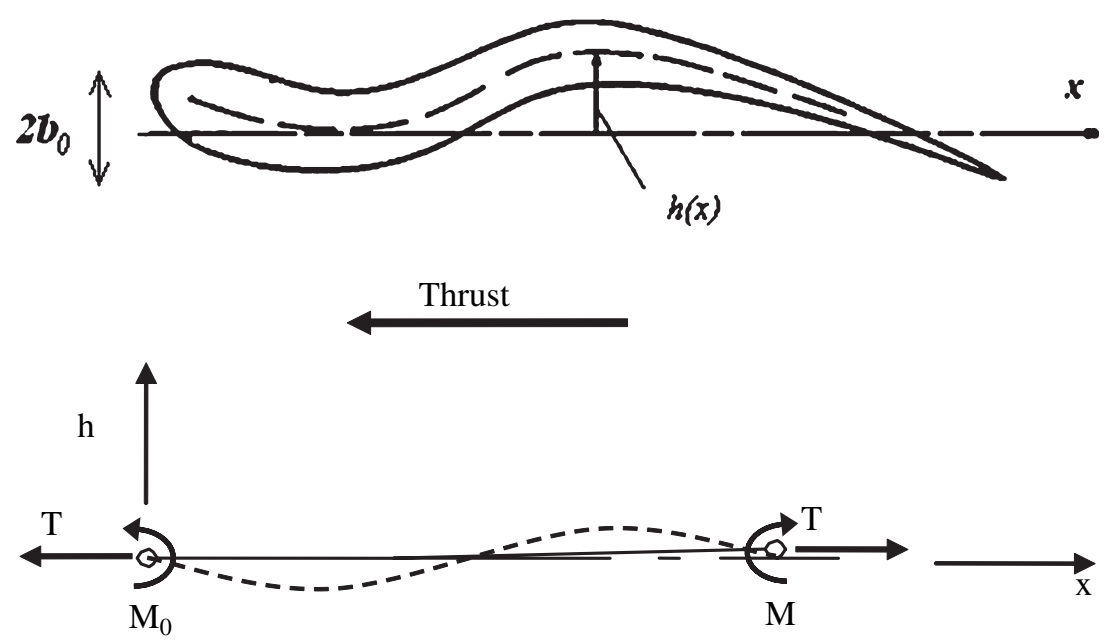

Figure 6: Inverse buckling of a fish: internal moment and tension-like forces $(T)$ induce reactive overall thrust force. 
small lateral displacements are considered. The solution of this equation consists of a waveform whose wavelength can be expressed through a Strouhal number based on the mechanical properties of the fish body. The predictable wavelength of the undulatory swimming, by means of the Strouhal number for the reverse buckling fish, agrees well with the apparently universal experimental value, observed by numerous investigators.

Consider a fish undergoing a steady-state undulatory swimming. A balance of the forces and bending moments on the fish, seen as a buckling tapered column, gives as a result an ordinary second-order differential equation of the type given by eqn (4), if small lateral displacements are considered. The area moment of inertia depends on the cross-section shape, which in the case of a fish is difficult to describe as it does not correspond to a regular geometric form with a simple mathematical expression. Depending on the species, the cross-section shape of a fish has been modelled as circular or elliptical [15], so the area moment of inertia with respect to a central (vertical) axis is proportional to $x^{4}$. Therefore, the coefficient of the second term in eqn (4) is proportional to $1 / x^{4}$ and the general solution of this equation $[39,40]$ is given by sine and cosine functions of the reciprocal of $x$, multiplied by $x$ itself, i.e.

$$
h=x\left(A_{1} \cos \frac{m}{x}+A_{2} \sin \frac{m}{x}\right),
$$

with a waveform as in Fig. 7a. The general solution expressed through eqn (10) corresponds to the fourth power of $x$ in the area moment of inertia of a circular or elliptical form in eqn (4). Any other cross-section shape would give a different exponent for $x$, but the general solution of eqn (4) can always be expressed as Bessel functions [29]. Note the close resemblance of the waveform in Fig. 7a and those expressed through Bessel functions, with the observed lateral displacements of the lengthwise body portions of a fish (Fig $7 \mathrm{~b}$ and c) [3, 5, 42].

The solution given by eqn (10) for a homogeneous boundary condition at $x=0$ and any finite lateral displacement $h$ at a given fish length, is an oscillatory function characterized by a unique wavelength, with increasing amplitude. The wavelength of the tapered-like column of a fish can be obtained from the square root of $m$ in eqn (10), i.e.

$$
\lambda_{\mathrm{B}}=2 \pi \sqrt{m}=2 \pi \sqrt{\frac{C}{E}} .
$$

This result can be expressed through a fish buckling Strouhal number as defined in eqn (6),

$$
\mathrm{St}_{\mathrm{B}}=\frac{D}{\lambda_{\mathrm{B}}},
$$

where $D$ is a characteristic transverse dimension, i.e. the width of the buckling column. In this case, it is $2 b_{0}$, the maximum fish thickness. Combining eqns (11) and (12) and using $D=2 b_{0}$,

$$
\mathrm{St}_{\mathrm{B}}=\frac{D}{\lambda_{\mathrm{B}}}=\frac{2 b_{0}}{2 \pi \sqrt{\frac{C}{E}}}=\frac{b_{0}}{\pi} \sqrt{\frac{E}{C}} .
$$




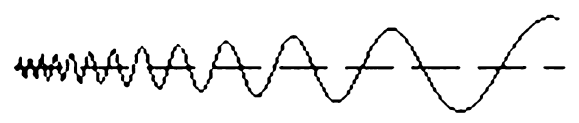

(a)
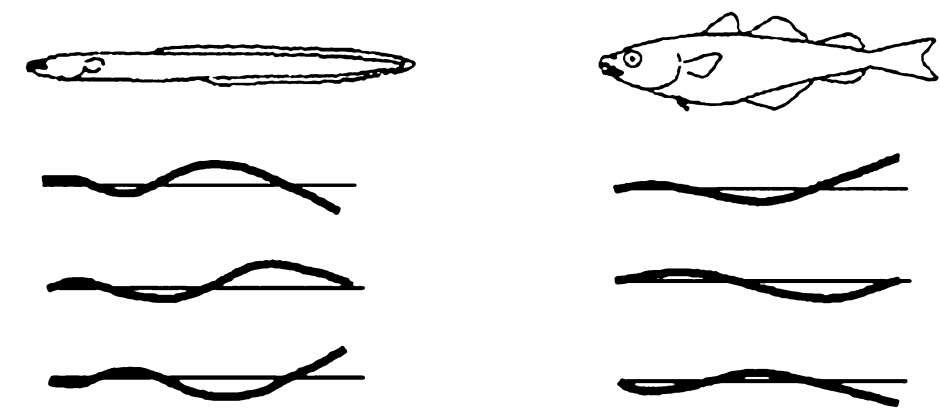

(b)

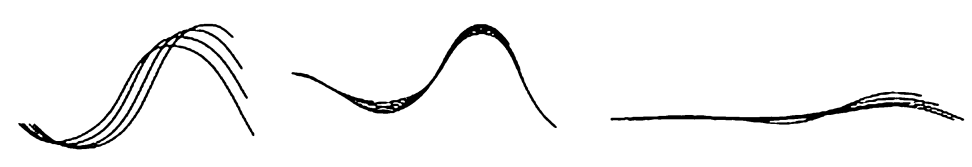

(c)

Figure 7: Undulatory waveforms: (a) eqn (10); (b) schematics of the extreme positions of the centre line of the body for eel and saithe species according to Wardle et al. [41] (adapted); (c) schematics of the fish undulatory swimming according to Videler [5] (adapted).

To validate the above-mentioned results in the context of fish swimming, appropriate experimental data is necessary. Although the archival literature extensively documents many aspects of fish life for a large variety of species, there are very few measurements on the mechanical and rheological properties of fish. Indeed, more high-quality data are urgently needed in this aspect of swimming fish, as reported by very few authors $[1,9,43,44]$. In this sense, let us take the morphological and stiffness properties reported by these authors of what seems to be the only published measured values of the mechanical stiffness properties of a fish obtained for a living and a laboratory killed sunfish specimen (Lepomis gibbosus, Lepomis macrochirus).

With an elastic modulus $E=180 \mathrm{~N} / \mathrm{m}^{2}$ (measured by McHenry et al. [9]), a fish half span $b_{0}=$ $0.0175 \mathrm{~m}$ (estimated by the authors this article from the morphometric data of Long et al. [1]) and a thrust force of $C=0.040 \mathrm{~N}$ (selected from the data obtained by Drucker and Lauder [44]), a buckling Strouhal number of $\mathrm{St}_{\mathrm{B}}=0.37$ is obtained. Testing other values of the above-mentioned physical parameters (for instance, $C$ in the range of $0.011-0.056 \mathrm{~N}$, and $b_{0}$ from 0.015 to 0.018 as reported by the same authors above), values for the Strouhal number in the range of 0.3-0.6 can be obtained. Table 1 shows these numbers together with the buckling Strouhal numbers estimated for solid and fluid columns, using the equations presented in this article.

These are highly significant and rewarding calculation results as they correspond to the mostly accepted experimental value for the Strouhal number of fish swimming $\mathrm{St}_{\mathrm{f}}=0.3$, based on the observed kinematic (oscillating) variables for many fish species, as reported by Triantafyllou et al. [7], 
Wolfgang et al. [17], and Taylor et al. [22]. They also correspond to the experimental values reported for flapping foils [22, as pointed out in the next section and included in Table 1.

The results presented herein are also close to the order of magnitude value of 0.5 for fluid columns estimated by Bejan [33] and calculated by Cervantes et al. [34].

\section{DISCUSSION AND CONCLUDING REMARKS}

A full picture of the undulatory swimming fish clearly emerges from the foregoing analysis. An incipient buckling Strouhal number, as an upstream precursor of the observed oscillating Strouhal number at the tail, can be predicted in a simple manner to characterize the undulating body. This is based on the analogy that appears between fish swimming and buckling columns (solid and fluid). The analogy at this stage is limited as it is based on small amplitude deflections. However, the important issue is that the model directly predicts the wavelength of the overall swimming movement of the fish. It corresponds to what Cheng et al. [15] regarded as the 'movement' approach, where a proper prediction of the lateral undulant displacement of the fish would be obtained as part of the solution, instead of the statement of the problem to establish the 'bending moment', assuming the waveform for the lateral displacements, as most researchers in this area do.

The analogy has two closely interconnected perspectives. On the one hand, a fish buckles and swims as a result of the unbalanced internal and external forces, composed mainly by the contraction and extension of muscles and the reaction to the external flow (drag and added mass). By generating undulatory waves that propagate from head to tail, and transferring momentum to the surrounding water, this hydrodynamic body produces thrust. Changing the shape and speed of these travelling waves, the fish controls its swimming speed. The complete and detailed study of the fish buckling during its steady-state rectilinear motion (with no acceleration) can be performed through a force and balance of bending moments, establishing equilibrium equations with an appropriate constitutive relationship.

In the present investigation, an analogue has been proposed for the steady-state fish swimming to a solid tapered column or a fluid column under axial compressive loading. An ordinary second-order differential equation is obtained whose solution consists of a single mode of oscillation: the onewavelength buckling response. This straightforward and simple procedure had not been proven up to now in fish locomotion analyses, as it required acknowledging this analogy as the incipient undulatory motion of the fish. If an accelerated motion could be considered as in very few investigations in this topic, then the dynamic balance equation takes the form of a wave equation (as mentioned at the beginning of this article) whose complete solution has not yet been reported in the open literature. It must be stressed at this point that the sinusoidal waveform for the fish undulation is invariably assumed, not predicted, in the published analyses.

On the other hand, as a result of the undulating swimming body, the travelling waves that emerge behind the rostral portion of the fish and grow in a controlled way along the entire body end as tail oscillations. This lateral motion has an important effect: the change in the rotation sense of the vortices system therein formed, transforms the dragging wake behind the fish into a thrusting penetrating jet, thus adding to the total impulsive force of the specimen. The experiments by Koochesfahani [21] for a flapping foil show that when a reverse Karman vortex street forms behind the foil, the vortex pattern is stable and the velocity profile has the form of a simple jet. This result has also been observed and considered by several authors in the fish swimming literature [17, 45, 46].

This effect corresponds well to the stability analysis predictions [7], where the optimal propulsion of an oscillating profile is obtained for the frequencies of maximum amplification of the average wake behind the profile, giving values of the oscillating Strouhal number in the range 0.25-0.35 (Table 1). 
Although the body buckling wavelength and the frequency of oscillations in the tail correspond to each other, there is a quantitative difference between the lateral displacement considered in the buckling response of the fish body and the amplitude of the oscillations at the tail that needs further clarification. That is, in the post-rostral region, where the incipient undulatory motion can be detected and modelled through the buckling theory, the transversal length scale that is employed in the buckling Strouhal number, is the fish body width, $2 b_{0}$, predicting Strouhal numbers in the range of 0.3-0.6, (eqn (10), Table 1). These values slightly increase along the fish body as the transverse displacement increases downstream, in less than $10 \%$ according to most of the reported experimental studies. In other words, the travelling wave continuously increases its amplitude in the downstream direction up to the tail where lateral flapping is observed. However, at the same time the wavelength increases in the same direction, perhaps in the same proportion, maintaining the Strouhal number within the same range of values. It is hard to identify the changes in the wavelength along the fish body, as most data in this respect in the published literature, is generally referred to the body length with a large variability among fish species and samples.

The process is much more complex than what has been described. It entails many aspects like the dynamic interactions between vortices, jets, and so on, as recently reviewed by Triantafyllou et al. [47]. But still the buckling description has three outstanding features: simplicity, predictability and physically grounded. The analogue points towards a more clear understanding of fish swimming and deserves to be considered and deepened.

\section{ACKNOWLEDGEMENTS}

This research has been partially supported by UNAM through the grant PAPIIT-IN115703 and by CONACYT through the grant SEP-2003-C02-43538.

\section{REFERENCES}

[1] Long, Jr., J.H., Adcock, B. \& Root, R.G., Force transmission via axial tendons in undulating fish: a dynamic analysis. Comp. Biochem. Phys. A, 133, pp. 911-929, 2002.

[2] Katz, S.L., Shadwick, R.E. \& Rapaport, H.S., Muscle strain histories in swimming milkfish in steady and sprinting gaits. J. Exp. Biol., 202, pp. 529-541, 1999.

[3] Hess, F. \& Videler, J.J., Fast continuous swimming of saithe (Pollachius virens): a dynamic analysis of bending moments and muscle power. J. Exp. Biol., 109, pp. 229-251, 1984.

[4] Cheng, J.-Y., Zhuang, L.-X. \& Tong, B.-G., Analysis of swimming three-dimensional waving plates. J. Fluid Mech., 232, pp. 341-355, 1991.

[5] Videler, J.J., Fish Swimming, Chapman and Hall: London, 1993.

[6] Wu, T.Y., Mathematical biofluiddynamics and mechanophysiology. Math. Met. App. Sci., 24, pp. 1541-1564, 2001.

[7] Triantafyllou, G.S., Triantafyllou, M.S. \& Grosenbaugh, M.A., Optimal thrust development in oscillating foils with application to fish propulsion. J. Fluids Struct., 7, pp. 205-224, 1993.

[8] Long, Jr., J.H., McHenry, M.J. \& Boetticher, N.C., Undulatory Swimming: how traveling waves are produced and modulated in sunfish (Lepomis Gibbosus). J. Exp. Biol., 192, pp. 129-145, 1994.

[9] McHenry, M.J., Pell, C.A. \& Long, Jr., J.H., Mechanical control of swimming speed: stiffness and axial wave form in undulating fish models. J. Exp. Biol., 198, pp. 2293-2305, 1995.

[10] Carling, J., Williams, T.L. \& Bowtell, G., Self-propelled anguilliform swimming: simultaneous solution of the two-dimensional Navier-Stokes equations and Newton's laws of motion. $J$. Exp. Biol., 201, pp. 3143-3166, 1998. 
[11] Pedley, T.J. \& Hill, S.J., Large-amplitude undulatory fish swimming: fluid mechanics coupled to internal mechanics. J. Exp. Biol., 202, pp. 3431-3438, 1999.

[12] Rohr, J.J. \& Fish, F.E., Strouhal numbers and optimization of swimming by odontocete cetaceans. J. Exp. Biol., 207, pp. 1633-1642, 2004.

[13] Sfakiotakis, M., Lane, D.M. \& Davies, J.B.C., Review of fish swimming modes for aquatic locomotion. IEEE J. Oceanic Eng., 24 (2), pp. 237-252, 1999.

[14] Blight, A.R., The muscular control of vertebrate swimming movements. Biol. Rev., 52, pp. 181-218, 1977.

[15] Cheng, J.-Y., Pedley, T.J. \& Altringham, J.D., A continuous dynamic beam model for swimming fish. Philos. Trans. R. Soc. London B, 353, pp. 981-997, 1998.

[16] Liu, H. \& Kawachi, K., A numerical study of undulatory swimming. J. Comp. Phys., 155, pp. 223-247, 1999.

[17] Wolfgang, M.J., Anderson, J.M., Grosenbaugh, M.A., Yue, D.K.P \& Triantafyllou, M.S., Nearbody flow dynamics in swimming fish. J. Exp. Biol., 202, pp. 2303-2327, 1999.

[18] Murray, M.M. \& Howle, L.E., Spring stiffness influence on an oscillating propulsor. J. Fluids Struct., 17, pp. 915-926, 2003.

[19] Jones, K.D. \& Platzer, M.F., Numerical computation of flapping-wing propulsion and power extraction. Paper AIAA-97-0826, 35th Aerospace Sciences Meeting \& Exhibit, Reno, NV, 6-10 January 1997.

[20] Streitlien, K. \& Triantafyllou, G.S., On thrust estimates for flapping foils. J. Fluids Struct., 12, pp. 47-55, 1998.

[21] Koochesfahani, M., Vortical patterns in the wake of an oscillating airfoil. AIAA J., 27(9), pp. 1200-1205, 1989.

[22] Taylor, G.K., Nudds, R.L. \& Thomas, A.R.L., Flying and swimming animals cruise at a Strouhal number tuned for high power efficiency. Nature, 425, pp. 707-711, 2003.

[23] Fung, Y.C., Biomechanics, Springer-Verlag: Berlin and New York, 1981.

[24] Long, Jr., J.H., Koob-Emunds, M., Sinwell, B. \& Koob, T.J., The notochord of hagfish Myxine glutinosa: visco-elastic properties and mechanical functions during steady swimming. J. Exp. Biol., 205, pp. 3819-3831, 2002.

[25] Shanley, F.R., Strength of Materials. McGraw-Hill Book Co.: New York, 1965.

[26] Li, G.Q. \& Li J.J., A tapered Timoshenko-Euler beam element for analysis of steel portal frames. J. Construct. Steel Res., 58, pp. 1531-1544, 2002.

[27] Vogel, S., Cat's Paws and Catapults, Norton: New York, 1998.

[28] Bejan, A., Shape and Structure, from Engineering to Nature, Cambridge University Press: Cambridge, UK, 2000.

[29] Gere, J.M. \& Carter, W.O., Critical buckling loads for tapered columns. ASCE J. Struct. Div., pp. 1-11 February 1962.

[30] Banerjee, J.R. \& Williams, F.W., Exact Bernoulli-Euler static stiffness matrix for a range of tapered beam-columns. Int. J. Numer. Meth. Eng., 23, pp. 1615-1628, 1986.

[31] Biot, M.A., Theory of viscous buckling of multilayered fluids undergoing finite strain. Phys. Fluids, 7, pp. 855-861, 1964.

[32] Taylor, G.I., Instability of jets, threads and sheets of viscous fluids. Proc. of the 12th Int. Cong. on Applied Mechanics, Stanford 1968, Springer-Verlag: Berlin, pp. 382-388, 1969.

[33] Bejan, A., Buckling flows: a new frontier in fluid mechanics. Annual Reviews in Numerical Fluid Mechanics and Heat Transfer, ed. T.C. Chawla, Hemisphere Pub. Co.: Washington, pp. 262-304, 1987.

[34] Cervantes, J.G., Solorio, F.J. \& Méndez, F., On the buckling and the Strouhal law of fluid columns: the case of turbulent jets and wakes. J. Fluid Struct., 17, pp. 1203-1211, 2003. 
[35] Cruickshank, J.O. \& Munson, B.R., Viscous fluid buckling of plane and axisymmetric jets. J. Fluid Mech., 113, pp. 221-239, 1981.

[36] Cervantes, J. \& Goldschmidt, V.W., The apparent flapping motion of a turbulent plane jet. Further experimental results. J. Fluids Eng., 103(1), pp. 119-126, 1981.

[37] Roshko, A., On the drag and shedding frequency of two-dimensional bluff bodies. NACA TN3169, 1954.

[38] Cervantes, J. \& Solorio, F., Entropy generation in a plane turbulent oscillating jet. Int. J. Heat Mass Transfer, 45, pp. 3125-3129, 2002.

[39] Simmons, G.F., Differential Equations, McGraw-Hill Book Co.: New York, 1972.

[40] Polyanin, A.D. \& Zaitsev, V.F., Handbook of Exact Solutions for Ordinary Differential Equations, CRC Press: Boca Raton, FL, 2002.

[41] Wardle, C.S., Videler, J.J. \& Altringham, J.D., Tuning in to fish swimming waves: body form, swimming mode and muscle function. J. Exp. Biol., 198, pp. 1629-1636, 1995.

[42] Müller, U.K., Van Den Heuvel, B.L.E., Stamhuis, E.J. \& Videler, J.J., Fish foot prints: morphology and energetics of the wake behind a continuously swimming mullet (chelon labrosus risso). J. Exp. Biol., 200, pp. 2893-2906, 1997.

[43] Drucker, E.G. \& Lauder, V.D., Locomotor forces on a swimming fish: three-dimensional vortex wake dynamics quantified using digital particle image velocimetry. J. Exp. Biol., 202, pp. 2393-2412, 1999.

[44] Drucker, E.G. \& Lauder, V.D., A hydrodynamic analysis of fish swimming speed: wake structure and locomotor force in slow and fast labriform swimmers. J. Exp. Biol., 203, pp. 2379-2393, 2000.

[45] Cheng, J.-Y. \& Chahine, G.L., Computational hydrodynamics of animal swimming: boundary element method and three-dimensional vortex wake structure. Comp. Biochem. Phys. Part A, 131, pp. 51-60, 2001.

[46] Triantafyllou, M.S., Triantafyllou, G.S. \& Yue, D.K.P., Hydrodynamics of fishlike swimming. Ann. Rev. Fluid Mech., 32, pp. 33-53, 2000.

[47] Triantafyllou, M.S., Techet, A.H. \& Hover, F.S., Review of experimental work in biomimetic foils. IEEE J. Oceanic Eng., 29(3), pp. 585-594, 2004. 